\title{
Engineering Demand for Smart Alarm Management in Large-Scale Industrial Processes
}

\author{
Fan Yang* \\ Tsinghua National Laboratory for Information Science and Technology, Department of Automation, Tsinghua University, Beijing 100084, China
}

Automation or control systems, such as Distributed Control Systems (DCSs) and Programmable Logic Controllers (PLCs) are commonly used in industries to help operators monitor and control the situation of the process. In order to detect abnormal situations and notify operators promptly, alarms are configured in the system to reflect faulty states of specific variables. Alarm management has attracted a lot of attention in industries because there are often too many poorly configured alarms that distract operators, leading to low efficiency of alarm systems. Special techniques are needed to optimize the alarm settings, namely, smart alarm management.

In an industrial process, when the process deviates from its normal situation, alarms are commonly used as warnings or alerts for operators that some actions should be taken immediately to fix the problem. If the root cause is found correctly and tackled promptly, then the process can return to its normal situation. However, if operators cannot identify the real root cause or cannot take right actions to tackle the problem in a short period of time, some terrible consequences may occur, which sometimes brings out accidents or catastrophes. Currently, emergency shot-down systems are always configured for large-scale processes, which serve as the last effort to prevent severe accidents, but it still brings out economic loss because of the unplanned shutdown. Therefore, alarm management is very important and should assist operators recognize the real fault. Ideally, one fault should trigger one exclusive alarm so that the operator can easily obtain accurate information from the alarm. In reality, however, there are too many nuisance alarms including chattering or repeated alarms.

There are some standards used as references for the alarm system design. In the typical EEMUA191 (version 2) [1] and ISA18.2 [2] standards, it is suggested that there should be no more than 6 alarms per hour per operator, which assumes that each operator needs 10 minutes to tackle one alarm in average. Currently there are various techniques aiming at reducing redundant alarms. For a single alarm design, an optimal filter, time delay, and deadband can be used to reduce chattering alarms effectively [3]. If this is perfectly conducted, $80 \%$ of the redundant alarms can be removed [4]. However, alarms are just symptoms of abnormal situations, and some abnormal situations can raise more than one alarms; thus multivariate alarm analysis is needed, where process knowledge, process data, and alarm data should be integrated to obtain the relationship between different alarms [4]. First, we can find related alarms in historical alarm data by statistical and visualization methods $[5,6]$; then check the process knowledge with the help of process data for a causal relationship [7]. These multivariate alarms with interrelationship are often shown as alarm floods, in which a lot of alarms occur within a short period of time due to propagation. There are usually two types of approaches. Root cause analysis aims at finding the fault propagation paths [8]. Based on this, the sequential alarms can be inactivated if the root cause of the problem has been solved. The second method is alarm sequence pattern recognition [9], where both the alarm sequence and time factors are incorporated. If the newly-detected alarm pattern is the same or similar as a known pattern, the related alarms can be grouped and reduced to one alarm.
In addition to the appropriate setting of alarms to deliver as few alarms as possible, we should also deliver accurate, plentiful, and constructive information to operators. Thus a well-designed alarm management system should not only notify the operator there is something wrong, but also guide the operator what may be the root cause and what action may be effective. Therefore, the smart alarm management system can be associated with an operator guide system. In the display of alarm events, there should be possible causes and suggested actions. Although the information provided may not be exclusive, some options can be provided to the operator with probabilities. This has not been realized by most of the researchers and practitioners.

Smart alarm management, as an emerging area, is a typical application-oriented interdisciplinary research area. We should go beyond the standards to dig out the useful information from the process knowledge and historical data.

\section{Acknowledgments}

The author would also thank Profs. Tongwen Chen and Sirish L. Shah and their group members for collaboration on alarm management. Financial aid is also appreciated from the National Natural Science Foundation of China (No. 60904044) and Tsinghua National Laboratory for Information Science and Technology Crossdiscipline Foundation.

\section{References}

1. Engineering Equipment and Materials Users Association (2007) Alarm Systems - A Guide to Design, Management and Procurement, $2^{\text {nd }}$ Edn, EEMUA.

2. International Society of Automation (2009) Management of Alarm Systems for the Process Industries, ANSI/ISA-18.2-2009.

3. Izadi I, Shah SL, Shook DS, Kondaveeti SR, and Chen T (2009) A Framework for Optimal Design of Alarm Systems. Proceedings of the 7th IFAC SAFEPROCESS, Barcelona, Spain, 651-656.

4. Izadi I, Shah SL, Chen T (2010) Effective resource utilization for alarm management. Proceedings of the $49^{\text {th }}$ IEEE Conference on Decision and Control (CDC). Atlanta, GA, USA, 6803-6808.

5. Kondaveeti SR, Izadi I, Shah SL, Black T (2010) Graphical representation of industrial alarm data. Proceedings of the $11^{\text {th }}$ IFAC Symposium on Analysis, Design and Evaluation of Human-Machine Systems, Valenciennes, France, 11.

6. Yang F, Shah SL, Xiao D, Chen T (2012) Improved correlation analysis and visualization of industrial alarm data. ISA Trans 51: 499-506.

7. Yang F, Shah SL, Xiao D (2012) International Journal of Applied Mathematics and Computer Science. Appl Numer Math 22: 41-53.

*Corresponding author: Fan Yang, Department of Automation, Tsinghua University, Beijing 100084, China, E-mail: yangfan@tsinghua.edu.cn

Received July 23, 2012; Accepted July 26, 2012; Published July 30, 2012

Citation: Yang F (2012) Engineering Demand for Smart Alarm Management in Large-Scale Industrial Processes. Adv Robot Autom 1:e106. doi:10.4172/21689695.1000e106

Copyright: (c) 2012 Yang F. This is an open-access article distributed under the terms of the Creative Commons Attribution License, which permits unrestricted use, distribution, and reproduction in any medium, provided the original author and source are credited. 
Citation: Yang F (2012) Engineering Demand for Smart Alarm Management in Large-Scale Industrial Processes. Adv Robot Autom 1:e106. doi:10.4172/2168-9695.1000e106

Page 2 of 2

8. Yang F, Xiao D (2012) Progress in root cause and fault propagation analysis of large-scale industrial processes. Journal of Control Science and Engineering 2012-Article ID 478373
9. Folmer J, Vogel-Heuser B (2012) Computing dependent industrial alarms for alarm flood reduction. Proceedings of the $9^{\text {th }}$ International Multi-Conference on Systems, Signals and Devices (SSD), Chemnitz, Germany 1-6. 